\title{
Antiparkinsonian Drug Reduction After Directional Versus Omnidirectional Bilateral Subthalamic Deep Brain Stimulation
}

\author{
Dávid Pintér, $M D, P^{1}$; Evelyn Járdaházi, $\mathrm{MA}^{1}$; István Balás, $\mathrm{MD}, \mathrm{PhD}^{2}$; \\ Márk Harmat, $\mathrm{MD}^{1}$; Tamás Makó, $\mathrm{MD}^{1}$; Annamária Juhász, $\mathrm{MD}^{1}$; \\ József Janszky, MD, DSc ${ }^{1,3}$; Norbert Kovács, MD, DSc ${ }^{1,3}$
}

\begin{abstract}
Background: Several pilot trials and the Clinical Evaluation of the Infinity Deep Brain Stimulation System (PROGRESS) study have found that directional stimulation can provide a wider therapeutic window and lower therapeutic current strength than omnidirectional stimulation.

Objective: We conducted a single-center, open-label, registry-based, comparative trial to test the hypothesis that directional stimulation can be associated with a greater reduction in the total daily dose of antiparkinsonian medications (ApMeds) than omnidirectional stimulation.

Materials and Methods: A total of 52 patients with directional and 57 subjects with omnidirectional bilateral subthalamic deep brain stimulation (STN-DBS) were enrolled. Preoperatively and 12 months postoperatively, the dose of different ApMeds, the number of tablets used daily, the severity of motor and nonmotor symptoms using the Movement Disorder Society-sponsored Unified Parkinson Disease Rating Scale, and the health-related quality of life (HRQoL) using the 39-item Parkinson's Disease Questionnaire (PDQ-39) were assessed.

Results: According to the changes in the levodopa equivalent daily dose, directional STN-DBS led to a $13 \%$ greater reduction in the total daily dose of ApMed. The $10.3 \%$ greater reduction in the dose of levodopa was the main contributor to this difference. The number of different ApMed types also could be decreased in a greater manner with directional stimulation. The improvement in the severity of motor and nonmotor symptoms was comparable; however, we detected a $15.8 \%$ greater improvement in the global HRQoL among patients with directional stimulation according to the changes in the summary index of the PDQ-39. The total electrical energy delivered per second was comparable between the groups at 12-month postoperative visit, whereas the amplitude of stimulation was significantly lower and the impedance was significantly higher with directional leads.

Conclusions: Directional programming can further increase the reduction in the total daily dose of ApMed after STN-DBS. In addition, directional stimulation can have additional beneficial effects on the global HRQoL. The greater reduction of ApMed doses did not require more energy-consuming stimulation with directional stimulation.
\end{abstract}

Keywords: Deep brain stimulation, directional stimulation, levodopa equivalent daily dose, Parkinson disease, therapeutic window

Conflict of Interest: Dávid Pintér was supported by the ÚNKP-20-4 New National Excellence Program of the Ministry for Innovation and Technology from the Source of the National Research, Development, and Innovation Fund for conducting this study and preparing the manuscript. Evelyn Járdaházi received a research grant from Abbott Laboratories for conducting this study. József Janszk received $<€ 1000$ consultation fees from Hungarian subsidiaries of UCB, Richter, and Gerot. Regarding this study, he did not receive any corporate funding. Norbert Kovács received $<€ 1000$ consultation fees from Hungarian subsidiaries

\footnotetext{
1 Department of Neurology, Medical School, University of Pécs, Pécs, Hungary;

2 Department of Neurosurgery, Medical School, University of Pécs, Pécs, Hungary; and

3 MTA-PTE Clinical Neuroscience MR Research Group, Pécs, Hungary
}

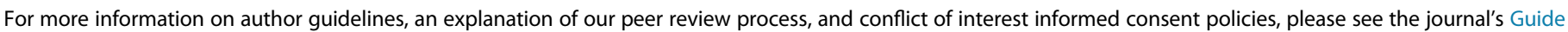
for Authors.

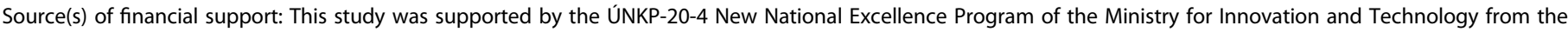

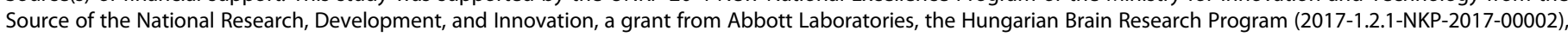

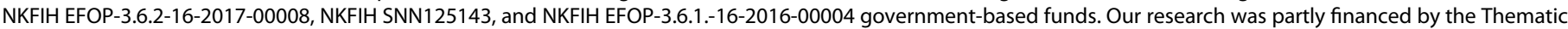

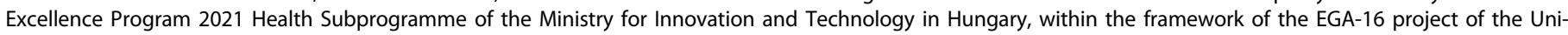
versity of Pécs. 
of Medtronic, Boehringer Ingelheim, Novartis, GlaxoSmithKline, UCB, Krka, and AbbVie. Regarding this study, he received a research grant from Abbott Laboratories for conducting the study and preparing the manuscript. The remaining authors reported no conflict of interest.

\section{INTRODUCTION}

Pharmaceutical treatment of advanced Parkinson disease (PD) can be highly challenging. At the later stages of the disease, the balancing of symptomatic improvement with drug-related complications is difficult or completely impossible. ${ }^{1}$ In addition, the long duration of antiparkinsonian pharmacotherapy and the frequently applied complex drug combinations ${ }^{2,3}$ impose a high economic burden on both the patients and the health care system. ${ }^{4,5}$ In advanced PD cases, subthalamic deep brain stimulation (STN-DBS) can be both a highly effective and cost-effective treatment option. ${ }^{6-14}$ The reduction in antiparkinsonian medication (ApMed) dosing and costs is considered as one of the most important contributors to the cost-effectiveness of this therapy. ${ }^{15-19}$

Recently, the technique of DBS has undergone a remarkable improvement. The development of directional leads is one of the most important milestones in this technical evolution. With this innovative technology, the direction of electric field spread can be focused to the targeted anatomical structure more precisely, which may offer more programming flexibility for optimizing the efficacy of stimulation and decreasing the risk for adverse events. $^{20,21}$ Single-center pilot studies that used directional leads for intraoperative test stimulation and examined the low number of cases with permanently implanted directional leads have found that the maximal stimulation amplitude that causes side effect can lie higher in single-segment activation mode, and the minimal stimulation amplitude required to achieve meaningful therapeutic benefit (therapeutic current strength [TCS]) also can be lower than with omnidirectional stimulation. Therefore, singlesegment activation can provide a wider therapeutic window (TW). ${ }^{22-26}$

The Clinical Evaluation of the Infinity Deep Brain Stimulation System (PROGRESS) study, an international, multicenter, prospective, crossover trial on the efficacy of the Abbott St Jude Infinity ${ }^{\mathrm{TM}}$ DBS system (Abbott Laboratories, Chicago, IL), has thus far been the largest scale investigation that compared the TWs provided by directional and omnidirectional programming during STN-DBS. It has been shown that directional stimulation increased the TW by $41 \%$, and the TW percentage (TW\%, the ratio of TW and TCS) also was $69 \%$ larger. In addition, to achieve these therapeutic benefits, 30\% less energy was required. According to these results, directional stimulation can provide more programming range with less energy. ${ }^{27,28}$

Based on the initial pilot trials and the first large-scale analysis of the PROGRESS study, single-segment activation seems to have better efficacy and safety profile (lower TCS, wider TW, and better TW\%) than omnidirectional stimulation. However, comparative data on the postoperative oral ApMed reduction after the implantation of directional and conventional leads are lacking. We assume that the wider TW provided by directional stimulation may enable a higher reduction in the dose of ApMeds. To test this hypothesis, we conducted a single-center, open-label, registrybased trial comparing the changes in the daily administered doses of ApMeds during directional vs omnidirectional bilateral STN-DBS in advanced PD.

\section{MATERIALS AND METHODS}

\section{Study Population and Protocol}

All study-related procedures were approved by the National Ethical Board (031037/2015/OTIG and OGYÉl/6916/2021).

This study was based on the prospective movement disorder registry of the Movement Disorder Unit of the Department of Neurology, University of Pécs. All patients in the registry who received directional leads (St Jude Medical Infinity ${ }^{\mathrm{TM}} 6170$ 8-Channel Directional Lead, Abbott Laboratories) in the subthalamic nuclei bilaterally for PD were included (directional stimulation group). Subsequently, from the same registry, a semiautomated software selected a matching group of patients with PD who underwent bilateral omnidirectional (Medtronic 3389 Quadripolar Omnidirectional Lead, Medtronic, Minneapolis, MN) STN-DBS implantation (omnidirectional stimulation group) based on demographic characteristics (eg, age, sex, and education), disease-specific data including type of PD, duration of disease, comorbidities, baseline (BL) rating scales (Movement Disorders Society-sponsored Unified Parkinson Disease Rating Scale [MDS-UPDRS] and the 39-item Parkinson's Disease Questionnaire [PDQ-39]), and ApMeds. The patients were treated with DBS according to the approved local guidelines and the actual CE mark of the respective devices. To enable contemporaneous comparison between the groups and minimize period effect, almost all patients in the omnidirectional cohort underwent surgery after the implantation of directional leads started in our center, and implantations were performed almost in parallel in the two groups by the same neurosurgeon using the same technique. The determination of the optimal theoretical STN target was based on previously reported coordinates $(9-12 \mathrm{~mm}$ lateral [X coordinate], 1-3 $\mathrm{mm}$ posterior [Y coordinate], and 3-5 $\mathrm{mm}$ inferior [ $Z$ coordinate] to the midcommissural point). ${ }^{29}$ During the preoperative planning that was performed with the StealthStation ${ }^{\mathrm{TM}}$ surgical navigation system (Medtronic), the theoretical coordinates were individualized based on the cerebral anatomical characteristics of patients. The final postoperative lead locations and active contact level coordinates at 12 months postoperatively (M12) were determined using postoperative computed tomography images in the StealthStation ${ }^{\mathrm{TM}}$ system and considering the characteristics of implanted electrodes (eg, lead diameter, contact height, and spacing). The detailed inclusion and exclusion criteria for the prospective data set on which the analysis is based can be found in the Supplementary Data.

The included patients had scheduled and standard examinations at $\mathrm{BL}$ (within three months before the surgery) and M12. In addition to recording detailed demographic (eg, age, sex, and level of education), disease-specific (eg, duration of disease, type of PD, and ApMeds), and comorbidity-related data, detailed neurological and health-related quality of life (HRQoL) measures were assessed at each visit. Stimulation parameters also were recorded at M12.

\section{Assessed Scales}

The cognitive performance was measured by the Hungarian validated versions (7.1, 7.2, and 7.3) of the Montreal Cognitive 
Assessment (MoCA). ${ }^{30}$ The severity of PD was globally measured by the Hungarian validated version of the MDS-UPDRS. ${ }^{31}$ To evaluate the HRQoL, the Hungarian validated versions of the PDQ$39^{32}$ and the nondisease specific EuroQoL five-dimension fivelevel health questionnaire (EQ-5D-5L) ${ }^{33}$ were used.

All scales were assessed at both BL and M12. All preoperative examinations were performed in the "best" on-medication state, whereas all postoperative measures were obtained in the medication-on and stimulation-on state.

\section{Primary End points}

The main outcome measures of the study included the differences in the change in the levodopa equivalent daily dose (LEDD) representing the total daily dose of all ApMeds ${ }^{34}$ from BL to M12, the number of patients receiving monotherapy in oral medication after STN-DBS, the number of subjects not receiving levodopa after STN-DBS, the number of tablets of the required daily ApMeds, and the number of different antiparkinsonian drug types after STN-DBS between the directional and omnidirectional stimulation groups.

\section{Secondary End points}

In this study, we also compared the level of changes in the severity of motor symptoms measured by Parts II and III of the MDS-UPDRS, the severity of nonmotor symptoms assessed by Part I of the MDS-UPDRS, the severity of motor complications evaluated by Part IV of the MDS-UPDRS, and the HRQoL measured by the summary index of the PDQ-39 and the EQ-5D-5L from BL to M12 between the directional and omnidirectional stimulation groups.

\section{Statistical Analysis}

Data were analyzed retrospectively. To test normality, the Kolmogorov-Smirnov test was used. Because most data from the applied scales followed the normal distribution, mean and SD values were calculated. For continuous data, independent $t$-tests were used, whereas for nominal and ordinal data, $x^{2}$ tests were used. The level of statistical significance was set at 0.05 . All statistical analyses were performed using the IBM SPSS software package (version 24.0.2, IBM Inc, Armonk, NY).

\section{RESULTS}

A total of 57 patients were considered for inclusion in the directional stimulation group. In the omnidirectional stimulation group, based on an automated script-based selection method ensuring balanced BL characteristics, the number of subjects also was 57 . Of the directional stimulation group, three patients were excluded because ring mode was used at $\mathrm{M} 12$, and two subjects were excluded because of missing values in key data (MDS-UPDRS and PDQ-39); therefore, data of 52 patients could be finally analyzed in this group.

Sociodemographic, disease-specific, and medication-specific data of the two groups at BL are shown separately in Table 1. Almost all the BL characteristics were comparable, with only the rescue drug, the water-soluble levodopa usage, differing significantly. The coordinates of active contact levels on DBS electrodes also were comparable (Table 2).

Changes in the key variables from BL to M12 can be found in Table 3. In the directional stimulation group, the LEDD decreased from $1267.8 \pm 661.9 \mathrm{mg}$ to $525.1 \pm 400.4 \mathrm{mg}$, whereas in the omnidirectional stimulation group, there was a decrease in the LEDD from $1139.8 \pm 429.5 \mathrm{mg}$ to $619.9 \pm 406.2 \mathrm{mg}$. At M12, LEDD was comparable between the two groups $(p=0.223)$; however, the magnitude of decrease was significantly higher with directional stimulation $(-742.6 \pm 550.7 \mathrm{mg}$ vs $-519.9 \pm 376.9 \mathrm{mg}, p=0.015)$. This primary end point was met.

At M12, the levodopa equivalent dose (LED) for drugs containing levodopa was comparable between patients with directional and omnidirectional stimulation $(295.5 \pm 376.2 \mathrm{mg}$ vs $321.7 \pm 331.1 \mathrm{mg}$, $p=0.699$ ). However, the magnitude of decrease in the LED for levodopa was significantly higher in the directional stimulation group $(-606.2 \pm 416.6$ vs $-423.7 \pm 308.9, p=0.010)$. The LED for dopamine agonists and the magnitude of change in the LED for dopamine agonists did not differ significantly between the two groups (191.2 \pm 229.6 vs $254.4 \pm 228.4, p=0.153$ and $-51.8 \pm 231.8$ vs $-27.7 \pm 214.4$, $p=0.575$ regarding directional and omnidirectional stimulation, respectively). According to these results, the greater reduction in the LEDD in the directional stimulation group mainly resulted from the greater decrease in the daily used dose of levodopa.

The number of patients receiving oral antiparkinsonian monotherapy increased from $6(11 \%)$ to $31(59.6 \%)$ in the directional stimulation group from $B L$ to $M 12$, whereas this number increased from $3(5.3 \%)$ to $27(47.4 \%)$ among patients with omnidirectional stimulation. Although the number of patients treated with oral monotherapy at $\mathrm{M} 12$ was higher in the directional stimulation group, the difference did not reach the level of statistical significance $(p=0.201)$. Therefore, this primary end point was not met.

From $\mathrm{BL}$ to $\mathrm{M} 12$, the number of patients not receiving levodopa increased from 0 to $23(44.2 \%)$ among patients with directional stimulation, whereas this number increased from 0 to $17(29.8 \%)$ in the omnidirectional stimulation group. Although the number of patients not receiving levodopa was in the directional stimulation group, the difference did not reach the level of statistical significance $(p=0.119)$. Therefore, this primary end point was not met.

The number of tablets of oral ApMeds used daily decreased from $7.8 \pm 3.0$ to $4.0 \pm 2.7$ in the directional stimulation group from $B L$ to $M 12$, whereas this number decreased from $7.5 \pm 2.3$ to $4.8 \pm 2.8$ among patients with omnidirectional stimulation. At M12, the number of tablets used daily was comparable in the two groups ( $p=0.136$ ); therefore, this primary end point was not met either.

The number of different antiparkinsonian drug types decreased from $2.8 \pm 1.1$ to $1.4 \pm 0.7$ among patients with directional stimulation from $\mathrm{BL}$ to $\mathrm{M} 12$; this number decreased from $2.6 \pm 0.8$ to $1.6 \pm 0.7$ in the omnidirectional stimulation group. The number of different ApMed types was comparable between the two groups ( $p=0.099)$; therefore, this primary end point was not met. However, the magnitude of decrease was significantly higher in the directional stimulation group $(-1.3 \pm 1.0$ vs $-0.9 \pm 0.9, p=0.036)$. Preoperative and postoperative doses of different ApMed types can be found in the Supplementary Data Tables S1 and S2.

The secondary outcomes are summarized in Table 4. Regarding the different parts of the MDS-UPDRS, neither the postoperative values nor the postoperative changes from BL to M12 differed significantly between the groups. From $B L$ to $M 12$, the summary index of the PDQ-39 improved from $28.4 \pm 15.0$ points to $17.3 \pm$ 10.8 points in the directional stimulation group, whereas it decreased from $24.5 \pm 12.2$ points to $18.9 \pm 13.9$ points among patients with omnidirectional stimulation. Although the level of the HRQoL was comparable between the two groups at M12 ( $p=$ 0.529), the magnitude of improvement in the summary index of the PDQ-39 was significantly higher in the directional stimulation group $(-11.1 \pm 12.3$ vs $-5.7 \pm 11.6, p=0.028)$. 
Table 1. BL Characteristics of the Study Population.

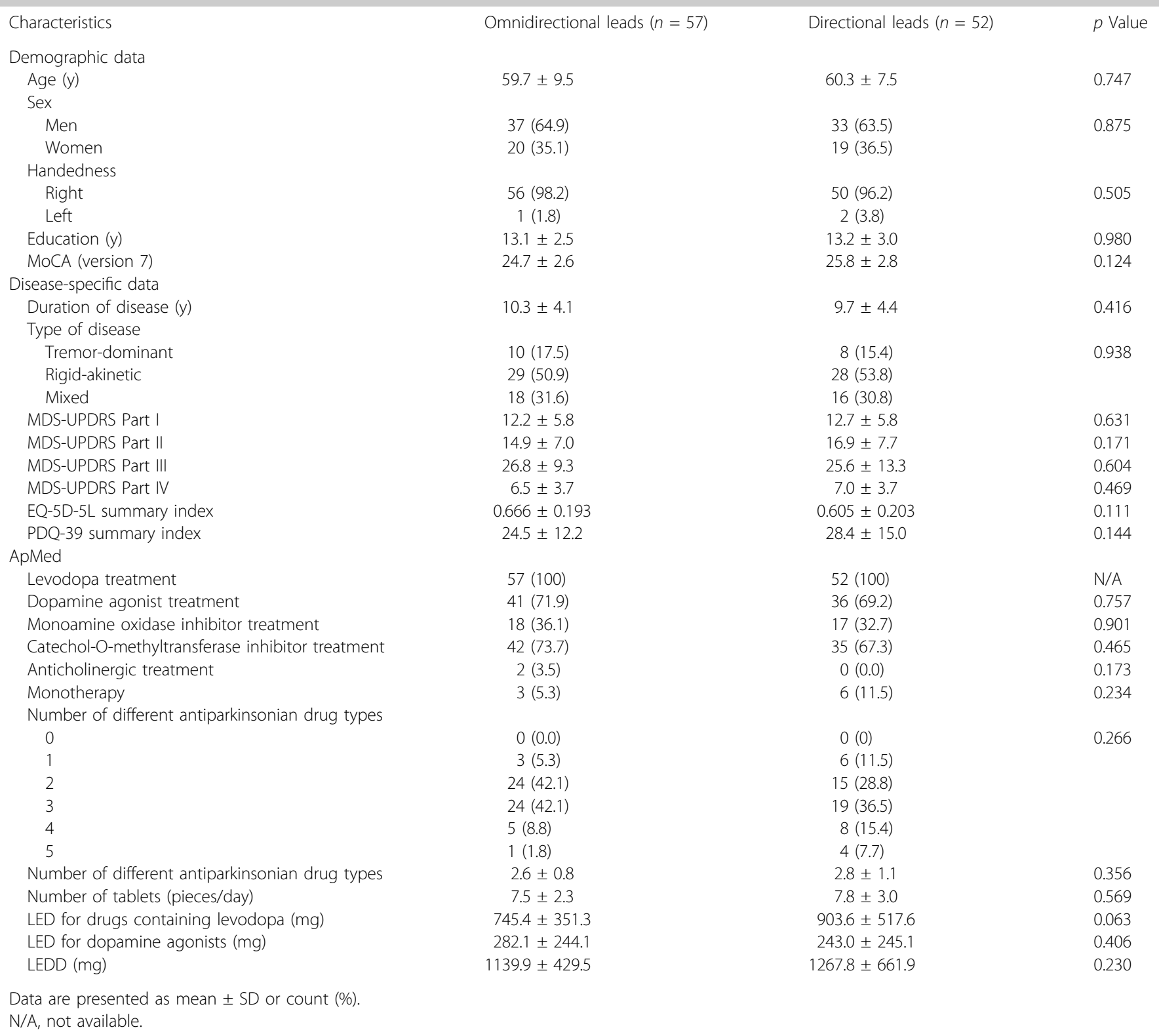

The total electrical energy delivered (TEED) per second calculated based on the method by Koss et $\mathrm{al}^{35}$ was comparable between the omnidirectional and directional stimulation groups
$(146.6 \pm 68.1 \mu \mathrm{J}$ vs $147.7 \pm 67.1 \mu \mathrm{J}, p=0.944)$ at M12. The amplitude of stimulation was significantly lower $(2.2 \pm 0.6 \mathrm{~mA}$ vs $2.6 \pm 1.0$ $\mathrm{mA}, p=0.022)$, whereas the impedance was significantly higher

Table 2. Coordinates of Active Contact Levels.

\begin{tabular}{|c|c|c|c|}
\hline Coordinates & Omnidirectional leads $(n=57)$ & Directional leads $(n=52)$ & $p$ Value \\
\hline \multicolumn{4}{|l|}{ Left lead } \\
\hline$X(m m)$ & $11.7 \pm 0.6$ & $11.6 \pm 1.1$ & 0.889 \\
\hline$Y(m m)$ & $2.7 \pm 0.5$ & $2.8 \pm 0.5$ & 0.837 \\
\hline \multicolumn{4}{|l|}{ Right lead } \\
\hline$X(\mathrm{~mm})$ & $12.3 \pm 0.6$ & $11.5 \pm 0.8$ & 0.120 \\
\hline$Y(\mathrm{~mm})$ & $2.3 \pm 0.7$ & $2.6 \pm 0.6$ & 0.491 \\
\hline
\end{tabular}


Table 3. The Primary Outcomes at the 12-Month Follow-Up Visit

Primary outcomes

LED for drugs containing levodopa (mg)

Change in the LED for drugs containing levodopa (mg)

LED for dopamine agonists (mg)

Change in the LED for dopamine agonists (mg)

LEDD (mg)

Change in the LEDD (mg)

Number of different antiparkinsonian drug types

Change in the number of different antiparkinsonian drug types

Number of tablets (pieces/day)

Change in the number of tablets (pieces/day)

Monotherapy

Change in the monotherapy status

Achieved

Unchanged

Lost

Levodopa treatment

Change in levodopa treatment

Stopped

Unchanged

Initiated

Dopamine agonist treatment

Change in dopamine agonist treatment

Stopped

Unchanged

Initiated

Monoamine oxidase inhibitor treatment

Change in monoamine oxidase inhibitor treatment

Stopped

Unchanged

Initiated

Catechol-O-methyltransferase inhibitor treatment

Change in catechol-O-methyltransferase inhibitor treatment

Stopped

Unchanged

Initiated

Anticholinergic treatment

Change in anticholinergic treatment

Stopped

Unchanged

Initiated
Omnidirectional leads $(n=57)$

$321.7 \pm 331.1$

$-423.7 \pm 308.8$

$254.4 \pm 228.4$

$-27.7 \pm 214.4$

$619.9 \pm 406.2$

$-519.9 \pm 376.9$

$1.6 \pm 0.7$

$-0.9 \pm 0.9$

$4.8 \pm 2.8$

$-2.6 \pm 3.1$

27 (47.4)

24 (42.1)

33 (57.9)

$0(0.0)$

$40(70.2)$

17 (29.8)

$40(70.2)$

$0(0.0)$

39 (68.4)

7 (12.3)

45 (78.9)

5 (8.8)

3 (5.3)

16 (28.1)

$40(70.2)$

1 (1.8)

29 (50.9)

14 (24.6)

42 (73.7)

1 (1.8)

$0(0.0)$

2 (3.5)

55 (96.5)

$0(0.0)$
Directional leads $(n=52)$

$p$ Value

$295.5 \pm 376.2$

0.699

$-606.2 \pm 416.6$

0.010

$191.2 \pm 229.6 \quad 0.153$

$-51.8 \pm 231.8 \quad 0.575$

$525.1 \pm 400.4 \quad 0.223$

$-742.6 \pm 550.7 \quad 0.015$

$1.4 \pm 0.7$

$-1.3 \pm 1.0$

$4.0 \pm 2.7$

$-3.7 \pm 2.9$

31 (59.6)

$26(50.0)$

25 (48.1)

1 (1.9)

29 (55.8)

23 (44.2)

29 (55.8)

$0(0.0)$

30 (57.7)

$10(19.2)$

38 (73.1)

$4(7.7)$

$0(0.0)$

$17(32.7)$

35 (67.3)

$0(0.0)$

19 (36.5)

0.099

0.036

0.136

0.063

0.201

0.376

0.119

0.246

0.605

18 (34.6)

32 (61.5)

2 (3.8)

$0(0.0)$

$0(0.0)$

52 (100)

$0(0.0)$

Data are presented mean \pm SD or count (\%).

N/A, not available.

$(1698.3 \pm 444.2 \Omega$ vs $1160.5 \pm 355.5 \Omega, p<0.001)$ with directional leads (Table 5).

There were no intracranial bleedings or infections in any of the included patients, and the frequency and severity of stimulationrelated side effects were comparable between the study groups.

\section{DISCUSSION}

Although an increasing body of research has revealed the superiority of directional stimulation over omnidirectional DBS, ${ }^{22-28}$ the effects of benefits provided by directional leads on antiparkinsonian pharmacotherapy have remained unknown. To address this question, we performed a single-center, open-label, registry-based, comparative study on the changes in the daily administered doses of ApMeds measured in LEDD during directional vs omnidirectional bilateral STN-DBS among patients with advanced PD.
One of the main findings of this study was that a greater reduction in the LEDD could be achieved with directional programming, which mainly resulted from the greater reduction in the daily used dose of medications containing levodopa. The magnitude of reduction in the total daily dose of ApMeds reached the $30 \%$ to $60 \%$ decrease that has been found in previous studies of STN-DBS ${ }^{15-17}$ in both study groups; however, the reductions in the total daily doses of ApMeds and levodopa were $13 \%$ ( $-58.6 \%$ vs $45.6 \%)$ and $10.3 \%(67.1 \%$ vs $56.8 \%)$, respectively, higher with directional stimulation. In addition, with directional stimulation, the oral antiparkinsonian treatment also could be simplified to a greater extent by reducing the numbers of different drug types and tablets used daily in a greater manner. These together not only may contribute to the higher cost-effectiveness of antiparkinsonian therapy but also may increase therapeutic adherence.

In line with the preliminary findings of the PROGRESS trial, ${ }^{27,28}$ this study also found no difference in the level of control of 
Table 4. The Secondary Outcomes at the 12-Month Follow-Up Visit.

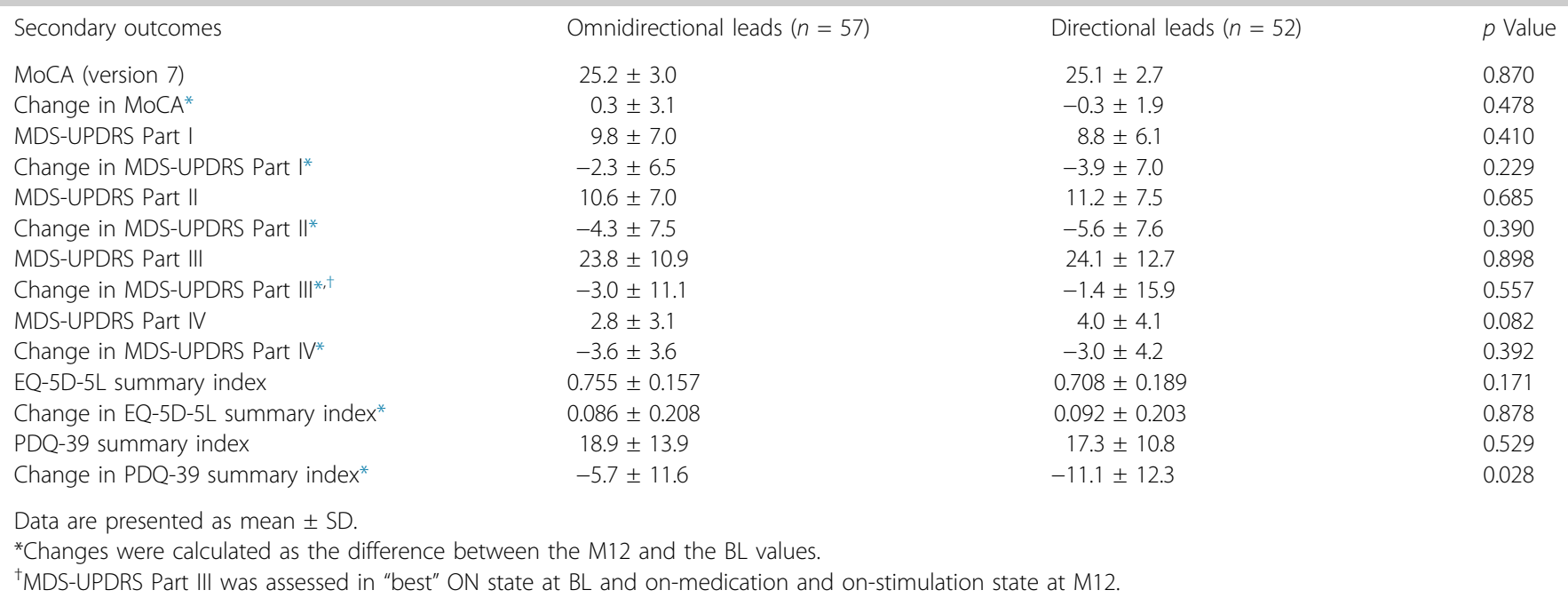

parkinsonian symptoms between directional and omnidirectional stimulation, despite the lower mean current strength used during directional stimulation. A possible explanation for this finding may be that directional leads can produce higher charge density with the same amplitude of current to the targeted brain structure and an increased volume of tissue activation compared with conventional omnidirectional leads. ${ }^{35}$ This increase in the activated volume of the targeted tissue can lead to a better symptomatic control, and as a result, lower dosages of additional oral medications may be needed to achieve the desired therapeutic effect. Furthermore, lower current thresholds for beneficial effects can prevent dose-limiting motor or nonmotor side effects resulting from the undesirable spread of stimulation into areas outside the target and can prevent an optimal outcome. In addition, reduced amplitude settings also can partially counterbalance the increased tissue impedance of smaller contacts, which may be relevant in terms of energy consumption. ${ }^{36}$

According to the summary index of the PDQ-39, we detected a $15.8 \%$ greater (39.1\% vs $23.3 \%$ ) improvement in the global HRQoL during directional STN-DBS. The change in the HRQoL was slightly inferior to that observed in previous studies of omnidirectional STN-DBS $(-24 \% \text { to } 38 \%)^{14}$ in the omnidirectional programming group, whereas the improvement exceeded that range with directional stimulation. The difference detected in the global HRQoL between the two groups was not just statistically significant but also clinically relevant, considering the minimal clinically important difference threshold for improvement established for the summary index of the PDQ-39 (4.72 points). ${ }^{32}$ In this study, directional stimulation provided the same symptomatic improvement and control as omnidirectional stimulation and greater improvement in the global HRQoL with less ApMeds, which may indicate the superior efficacy of directional vs omnidirectional stimulation.

Frequently applied complex drug combinations are among the main expenses of treatment in advanced PD. ${ }^{4,5}$ By reducing the dose of oral medications, STN-DBS can improve the costeffectiveness of antiparkinsonian therapy..$^{15,16,18,19}$ The PROGRESS study found that directional programming can be associated with lower energy consumption than omnidirectional stimulation. ${ }^{27,28}$ In this study, although directional stimulation was superior to omnidirectional stimulation in decreasing the total daily dose of oral ApMeds, the TEED per second was comparable. This means that the greater reduction of ApMed doses did not require more energy-consuming stimulation to achieve the desired symptomatic control. Furthermore, we detected a greater improvement in the global HRQoL that also has been considered as an important factor in previous analyses on the economic aspects of neurostimulation. ${ }^{37-39}$ Based on these findings, the use of directional leads may further improve the cost-effectiveness of DBS in PD.

During the interpretation of our findings, some limitations should be considered. First, patients were allocated to the treatment arms in a nonrandom fashion. In addition, the study is limited

Table 5. DBS Therapy Characteristics at the 12-Month Follow-Up Visit.

\begin{tabular}{|c|c|c|c|}
\hline Stimulation parameters & Omnidirectional leads $(n=57)$ & Directional leads $(n=52)$ & $p$ Value \\
\hline Amplitude (mA) & $2.6 \pm 1.0^{*}$ & $2.2 \pm 0.6$ & 0.022 \\
\hline Pulse width $(\mu s)$ & $62.8 \pm 9.9$ & $63.7 \pm 11.4$ & 0.725 \\
\hline Frequency $(\mathrm{Hz})$ & $146.5 \pm 24.9$ & $141.5 \pm 23.0$ & 0.399 \\
\hline TEED per second $(\mu J)$ & $146.6 \pm 68.1$ & $147.7 \pm 67.1$ & 0.944 \\
\hline
\end{tabular}


by its open-label and single-center design; our results might have been influenced by factors related specifically to the institution and/or the country. Because there is no unified strategy for programming, other movement disorder centers may use programming algorithms different from that used by our group. In our center, each patient undergoes monopolar review one month postoperatively. During this procedure, all segments are tested with standard parameters, namely, $60-\mu$ s pulse width and $130-\mathrm{Hz}$ frequency, and single segments are activated immediately after the monopolar review. If the outcomes justify it, single-segment activation is the default stimulation mode. During follow-up visits, if modifications to stimulation settings are needed, mainly the amplitude, occasionally the pulse width or the frequency, is adjusted instead of returning to ring mode. Strategic reduction of ApMeds after the initiation of DBS also may differ among centers. ${ }^{40}$ Our group prefers reducing the dose of levodopa in most of the cases and tries to achieve monotherapy with lowered doses of dopamine agonists. In addition, we tested more than one outcome; therefore, a type I error might have occurred that could be addressed with more in-depth analyses (eg, correction for multiple comparisons). ${ }^{41}$ Finally, because the study was based on the retrospective analysis of registry data, our findings provide class III evidence $^{42}$ on the difference in the reduction of ApMeds during omnidirectional vs directional STN-DBS.

In conclusion, compared with omnidirectional stimulation, directional STN-DBS can be associated with a greater reduction in the total daily dose of ApMeds, which mainly results from the reduction in the daily used doses of drugs containing levodopa. Therefore, it can be suggested that the use of directional leads that also have been found to have lower energy consumption may enable a more cost-effective antiparkinsonian treatment. Although the total daily dose of ApMeds can be reduced in a greater manner, the efficacy of directional DBS remains comparable with that of omnidirectional stimulation. Moreover, directional stimulation can lead to a greater improvement in the HRQoL of patients with PD. According to some previous findings, the reduced and simplified antiparkinsonian pharmacologic treatment and the improvement in the HRQoL can be sustainable even for years after the initiation of STN-DBS. ${ }^{43-45}$ Further longer-term studies should be conducted to decide whether observed differences in the level of antiparkinsonian drug reduction and the level of improvement in the HRQoL between directional and omnidirectional stimulation persist in the long term. Our findings support the suspected superiority of directional over omnidirectional stimulation from a previously unstudied aspect and can be useful for future efficacy and costeffectiveness analyses that are warranted to judge the clinical and economic relevance of our results.

\section{Authorship Statements}

Dávid Pintér and Norbert Kovács participated in all aspects of the research project and manuscript draft, review, and critique. Evelyn Járdaházi, Annamária Juhász, and Márk Harmat participated in the organization of the research project, the execution of the statistical analysis, and the review and critique of the manuscript. István Balás participated in the execution of the research project and the review and critique of the statistical analysis and the manuscript. Tamás Makó participated in the organization of the research project and the review and critique of the statistical analysis and the manuscript. József Janszky participated in the conception of the research project and the review and critique of the statistical analysis and the manuscript.

\section{How to Cite This Article}

Pintér D., Járdaházi E., Balás I., Harmat M., Makó T., Juhász A., Janszky J., Kovács N. 2023. Antiparkinsonian Drug Reduction After Directional Versus Omnidirectional Bilateral Subthalamic Deep Brain Stimulation.

Neuromodulation 2023; 26: 374-381.

\section{SUPPLEMENTARY DATA}

To access the supplementary material accompanying this article, visit the online version of Neuromodulation: Technology at the Neural Interface at www.neuromodulationjournal.org and at https:// doi.org/10.1016/j.neurom.2022.01.006.

\section{REFERENCES}

1. Aquino CC, Fox SH. Clinical spectrum of levodopa-induced complications. Mov Disord. 2015;30:80-89.

2. Oertel WH, Berardelli A, Bloem BR, et al. Late (complicated) Parkinson's disease. In: Gilhus N, Barnes PJ, Brainin M, eds. European Handbook of Neurological Management. Blackwell Publishing Ltd; 2011:237-267.

3. Aschermann Z, Kovács N, Komoly S. Continuous dopaminergic stimulation in Parkinson disease: possibilities in 2013. Article in Hu. Ideggyogy Sz. 2013;66:209-210.

4. Spottke $A E$, Reuter M, Machat $O$, et al. Cost of illness and its predictors for Parkinson's disease in Germany. Pharmacoeconomics. 2005;23:817-836.

5. Winter Y, von Campenhausen S, Reese JP, et al. Costs of Parkinson's disease and antiparkinsonian pharmacotherapy: an Italian cohort study. Neurodegener Dis. 2010;7:365-372.

6. Deuschl G, Schade-Brittinger C, Krack $P$, et al. A randomized trial of deep-brain stimulation for Parkinson's disease. N Engl J Med. 2006;355:896-908.

7. Timmermann $L_{\text {, Jain }} \mathrm{R}_{r}$ Chen $\mathrm{L}_{\text {, et }}$ al. Multiple-source current steering in subthalamic nucleus deep brain stimulation for Parkinson's disease (the VANTAGE study): a non-randomised, prospective, multicentre, open-label study. Lancet Neurol. 2015;14:693-701.

8. Weaver FM, Follett KA, Stern M, et al. Randomized trial of deep brain stimulation for Parkinson disease: thirty-six-month outcomes. Neurology. 2012;79:55-65.

9. Meissner W, Schreiter D, Volkmann J, et al. Deep brain stimulation in late stage Parkinson's disease: a retrospective cost analysis in Germany. I Neurol. 2005;252:218-223.

10. Valldeoriola F, Morsi O, Tolosa E, Rumià J, Martí MJ, Martínez-Martín P. Prospective comparative study on cost-effectiveness of subthalamic stimulation and best medical treatment in advanced Parkinson's disease. Mov Disord. 2007;22:21832191.

11. Tomaszewski KJ, Holloway RG. Deep brain stimulation in the treatment of Parkinson's disease: a cost-effectiveness analysis. Neurology. 2001;57:663-671.

12. Deli G, Aschermann Z, Ács P, et al. Bilateral subthalamic stimulation can improve sleep quality in Parkinson's disease. J Parkinsons Dis. 2015;5:361-368.

13. Fabbri M, Coelho M, Guedes LC, et al. Acute response of non-motor symptoms to subthalamic deep brain stimulation in Parkinson's disease. Parkinsonism Relat Disord. 2017:41:113-117.

14. Jiang J, Lo S, Tsai S, Chen S. A systematic review of the impact of subthalamic nucleus stimulation on the quality of life of patients with Parkinson's disease. Tzu Chi Med J. 2014:26:15-20.

15. Toft M, Dietrichs E. Medication costs following subthalamic nucleus deep brain stimulation for Parkinson's disease. Mov Disord. 2014;29:275-276.

16. Weaver FM, Stroupe KT, Cao L, et al. Parkinson's disease medication use and costs following deep brain stimulation. Mov Disord. 2012;27:1398-1403.

17. Kleiner-Fisman G, Herzog J, Fisman DN, et al. Subthalamic nucleus deep brain stimulation: summary and meta-analysis of outcomes. Mov Disord. 2006;21(suppl 14):S290-S304.

18. Hacker ML, Currie AD, Molinari AL, et al. Subthalamic nucleus deep brain stimu lation may reduce medication costs in early stage Parkinson's disease. J Parkinsons Dis. 2016;6:125-131.

19. Fehér G, Balás I, Komoly S, et al. Analysis of antiparkinsonian drug reduction after bilateral subthalamic deep brain stimulation. Article in Hu. Ideggyogy Sz. 2010;63:314-319. 
20. Volkmann J, Moro E, Pahwa R. Basic algorithms for the programming of deep brain stimulation in Parkinson's disease. Mov Disord. 2006;21(suppl 14):S284-S289.

21. Schüpbach WMM, Chabardes S, Matthies C, et al. Directional leads for deep brain stimulation: opportunities and challenges. Mov Disord. 2017;32:1371-1375.

22. Pollo C, Kaelin-Lang A, Oertel MF, et al. Directional deep brain stimulation: an intraoperative double-blind pilot study. Brain. 2014;137:2015-2026.

23. Contarino MF, Bour $L J$, Verhagen $R$, et al. Directional steering: a novel approach to deep brain stimulation. Neurology. 2014;83:1163-1169.

24. Steigerwald F, Müller L, Johannes S, Matthies C, Volkmann J. Directional deep brain stimulation of the subthalamic nucleus: a pilot study using a novel neurostimulation device. Mov Disord. 2016;31:1240-1243.

25. Dembek TA, Reker P, Visser-Vandewalle V, et al. Directional DBS increases sideeffect thresholds-a prospective, double-blind trial. Mov Disord. 2017;32:13801388.

26. Rebelo P, Green AL, Aziz TZ, et al. Thalamic directional deep brain stimulation for tremor: spend less, get more. Brain Stimul. 2018;11:600-606.

27. Schnitzler A, Mir P, Brodsky MA, et al. Directional versus omnidirectional deep brain stimulation: results of a multicenter prospective blinded crossover study [abstract]. Mov Disord. 2020. Accessed May 10, 2021. https://www.mdsabstracts.org/abstract/ directional-versus-omnidirectional-deep-brain-stimulation-results-of-a-multicenterprospective-blinded-crossover-study/

28. Directional deep brain stimulation linked to wider therapeutic window in Parkinson disease. Neurology Live. September 12, 2020. Accessed May 10, 2021. https:// www.neurologylive.com/view/therapeutic-window-superior-with-directional-dbsover-omnidirectional-stimulation

29. Dormont D, Seidenwurm D, Galanaud D, Cornu P, Yelnik J, Bardinet E. Neuroimaging and deep brain stimulation. AJNR Am J Neuroradiol. 2010;31:15-23.

30. Lucza T, Karádi K, Kállai J, et al. Screening mild and major neurocognitive disorders in Parkinson's disease. Behav Neurol. 2015;2015:983606.

31. Horvath $K$, Zsuzsanna A, Acs P, et al. Validation of the Hungarian MDS-UPDRS: why do we need a new Parkinson scale? Article in Hu. Ideggyogy Sz. 2014;67:129-134.

32. Horváth K, Aschermann Z, Kovács M, et al. Changes in quality of life in Parkinson's disease: how large must they be to be relevant? Neuroepidemiology. 2017;48:1-8
33. Szende A, Németh R. Health-related quality of life of the Hungarian population. Article in Hu. Orv Hetil. 2003;144:1667-1674.

34. Tomlinson CL, Stowe R, Patel S, Rick C, Gray R, Clarke CE. Systematic review of levodopa dose equivalency reporting in Parkinson's disease. Mov Disord. 2010;25:2649-2653.

35. Koss AM, Alterman RL, Tagliati M, Shils JL. Calculating total electrical energy delivered by deep brain stimulation systems. Ann Neurol. 2005;58:168.

36. Butson C, Venkatesan L. Comparison of neural activation between standard cylindrical and novel segmented electrode designs. Poster presented at: International Congress of the Movement Disorders Society; June 8-12, 2014; Stockholm, Sweden.

37. Dams J, Siebert U, Bornschein B, et al. Cost-effectiveness of deep brain stimulation in patients with Parkinson's disease. Mov Disord. 2013;28:763-771.

38. Eggington S, Valldeoriola F, Chaudhuri KR, Ashkan K, Annoni E, Deuschl G. The cost-effectiveness of deep brain stimulation in combination with best medical therapy, versus best medical therapy alone, in advanced Parkinson's disease. J Neurol. 2014;261:106-116.

39. Pietzsch JB, Garner AM, Marks WJ Jr. Cost-effectiveness of deep brain stimulation for advanced Parkinson's disease in the United States. Neuromodulation. 2016;19:689-697.

40. Fasano A, Appel-Cresswell S, Jog M, et al. Medical management of Parkinson"s disease after initiation of deep brain stimulation. Can J Neurol Sci. 2016;43:626-634.

41. Chen SY, Feng $Z$, Yi X. A general introduction to adjustment for multiple comparisons. J Thorac Dis. 2017;9:1725-1729.

42. Sackett DL. Rules of evidence and clinical recommendations on the use of antithrombotic agents. Chest. 1989;95(suppl 2):2S-4S.

43. Zibetti M, Merola A, Rizzi L, et al. Beyond nine years of continuous subthalamic nucleus deep brain stimulation in Parkinson's disease. Mov Disord. 2011;26:23272334.

44. Schüpbach WM, Maltête D, Houeto JL, et al. Neurosurgery at an earlier stage of Parkinson disease: a randomized, controlled trial. Neurology. 2007;68:267-271.

45. Schuepbach WM, Rau J, Knudsen K, et al. Neurostimulation for Parkinson's disease with early motor complications. N Engl J Med. 2013;368:610-622. 